\title{
An innovative Erasmus Mundus Master program in Color in Informatics and Media Technology
}

\author{
Alain Trémeau* , Jon Hardeberg ${ }^{\dagger}$, Javier Hernandez-Andrès ${ }^{\ddagger}$, Juan Luis Nieves ${ }^{\ddagger}$, Jussi Parkkinen ${ }^{\S}$ \\ *Coordinator of master CIMET, Université Jean Monnet, Saint-Etienne, France \\ ${ }^{\dagger}$ Gjovik University College, Norway, ${ }^{\ddagger}$ University of Granada, Spain, ${ }^{\S}$ University of Eastern Finland \\ Email: alain.tremeau@univ-st-etienne.fr
}

\begin{abstract}
RESUME : The main objective of this paper is to identify and disseminate good practice in quality assurance and enhancement as well as in teaching and learning at master level. This paper focuses on the experience of the Erasmus Mundus Master program CIMET (Color in Informatics and Media Technology). Amongst topics covered, we discuss the adjustments necessary to a curriculum designed for excellent international students and their preparation for a global labor market.
\end{abstract}

Mots clés (Kewords): good practice, internal quality systems, innovations in curriculum design, teaching and learning, challenges of internationalization.

\section{INTRODUCTION.}

Since 2008, the CIMET consortium composed of University Jean Monnet (France), Gjøvik University College (Norway), University of Eastern Finland, and University of Granada (Spain) offers a two-year master course entitled Color in Informatics and Media Technology, see for more details [1].

\section{DESCRIPTION AND OBJECTIVES OF THE MASTER}

The CIMET Master program is broadly interdisciplinary and the course curriculum covers innovative areas such as color, photonics, computer vision and imaging science, computer science and multimedia technology. The program objective is to educate students in advanced methodologies and models in computational color science. With a perfect mix of relevant theoretical and practical knowledge, CIMET post-graduates will be in the position to engage in further academic research or join major companies in the IT industry. CIMET offers three areas of specialization: Color Imaging Science, Spectral Color Science and Media Technology. Those fields are increasingly emergent, rapidly evolving, and have an increasing impact on the Society of Information Technologies. They require more and more specialists and specialized competencies.

The CIMET Course takes place over four semesters.

- Semester 1 is dedicated to fundamental courses and takes place either at University Jean-Monnet (France) or University of Granada (Spain).

- During Semester 2, students start their first specialization according to their specific interests either at University Jean Monnet (France) or University of Granada (Spain).

- Over Semester 3, students do a second specialization either at University of Eastern Finland or Gjøvik University College (Norway).
- Semester 4 is dedicated to the Master Thesis. From January onward, students join a laboratory from one of the consortium universities and undertake their Master Thesis project.

Mobility is inherent to the CIMET program and is organized so that it brings added value to the overall students' training scheme. Mobility is also practiced by guest teachers and guest researchers belonging to the consortium or from outside the consortium.

The courses aim to develop:

- a systematic understanding of knowledge in color science, computer vision, image science and media technology, informed by a critical awareness of current problems and recent research;

- a framework within which students can continue to develop the knowledge and intellectual skills that will enable them to deal with the optical, imaging and media technologies of the future;

- a conceptual understanding that enables students to critically evaluate past and present research in specialized fields ;

- a comprehensive understanding of techniques applicable to research in multidisciplinary fields, and a practical understanding of how such techniques are applied to interpret and get ahead of existing knowledge;

The objective is that students are able to:

- demonstrate an advanced knowledge and understanding of specializations at the forefront of discipline in technical informatics; - develop methods to test and to improve processes; - to develop software for image or vision processing;

- demonstrate an advanced knowledge of imaging processes, including capture and digitization, processing, and output on a range of media and devices, and originality in its application; - overcome the global nature of an imaging chain from the image building to its exploitation on both the physical and computational point of view; 
- conceive industrial systems and devices using optical, optoelectronic and computational techniques;

- critically evaluate their own work, identify weaknesses and propose alternative solutions;

- plan and conduct a self-managed program of research and enquiry ; - analyze a problem both in its technical and economical prospects;

- lead and coordinate a research project; - communicate the results of their work clearly to specialist and non-specialist audiences; -communicate in a scientific or business context in several languages.

The CIMET curriculum is intended for top level graduates in physics, optics, imaging science, computer science, mathematics, or any discipline pertaining to the quantitative description of color, with a fluent knowledge in spoken and written English. Courses are structured according to the ECTS with 120 credits acquired over four semesters of full-time study. The master is entirely taught in English.

Successful students are awarded Masters Degrees issues by the Universities they have been visiting during the two-year program. Depending on the chosen mobility scheme, they are awarded at minimum a Double Degree (since they are ought to study in at least two universities of the consortium) or a multiple Degree. The obtained Degrees are officially recognized at national levels and give full access to $\mathrm{PhD}$ study programs. On the top of awarded National Degrees successful students are delivered an Erasmus Mundus Diploma supplement stating the European nature of the Course. All successful students having the same curriculum receive the same Degree whatever their country of origin.

In 2008, twenty four students from 13 nationalities (such as India, China, Indonesia, Ethiopia, US, France, Germany, etc.) have been enrolled in this master program. In average around twenty students are enrolled each year in this master.

\section{A MASTER OF EXCELLENCE ACCRE- DITED BY THE EACEA}

The Master benefit from the seal of excellence of the Erasmus Mundus program. This accreditation responds to excellence criteria expected both for the teaching and learning and the recruitment of the students. The level of expectations of the selected students and their sharp interest for the program are much higher than in any other more "common" master. One demonstration of this is highly visible in the academic results of the students (when certain courses are pooled with other Master courses, as for example with national master courses, the results of CIMET students are systematically higher than their counterpart) and in their commitment to the course. In addition, end of semester questionnaires pertaining to courses and academics do systematically reveal any discrepancies between stu- dent expectations and teacher deliveries (English proficiency, excellence in the topic) and course structure.

One of the success elements of the program is the selection of highly motivated and excellent students. To demonstrate excellence, the following selection criteria have been set:

1. Excellence of the candidate: outstanding achievement in the applicant's BSc degree level in physics, optics, imaging science, computer science, or any discipline pertaining to the quantitative description of color, provided that the applicant can provide evidence of the necessary previous knowledge (i.e. a base of minimal competencies) particularly in the fields of physical/technical fundamentals, computer science fundamentals, and image analysis and signal processing fundamentals. The applicants must have awarded at least a $\mathrm{C}$ average on ECTS grading scale, or equivalent (e.g. a GPA $=3.2 / 4$ ), during their undergraduate studies. The CIMET selection committee reserves the right to moderate this criterion according to the prestige of the diploma or the University of origin of the student.

2. Language ability: teaching and examination being given in English, the candidates must demonstrate sound knowledge of the language (the requirement for competence in English is equivalent to TOEFL with at least 213 points (computer based)/550 points (paper based) or IELTS at grade 6.5, or a Cambridge Proficiency Certificate of English at least grade C). Knowledge of French, Spanish, Finnish, and Norwegian is not compulsory, but will be beneficial.

3. Student motivation: motivation to undertake the Masters and relevance to his/her professional development (explaining the application, the present situation, the choice of the second semester specialization, the interest in the degree, the intentions after this degree, ...).

The following documents are also requested:

1. A Curriculum Vitae (written in English) stating the main personal information (birth name, birth date, citizenship, address, ...) and the studies since high school (degrees, institutions, grades);

2. Copies of transcripts or other academic records (e.g. diploma supplement) that give proof on the undergraduate and first graduate studies, including credits and grades achieved in each single subject. Documents must be either in English or in original language accompanied by a certified English translation. Depending on the home countries, applicants may be asked to provide Certificates of Authenticity issued by recognized diplomatic or administrative authorities, and to send them by regular mail. 
3. Two letters of recommendation (written in English) from academic and/or industrial members.

4. A certificate of language proficiency in English (see above);

5. A detailed list of research project and publications, if they are mentioned in the Vitae.

One of the unexpected findings is that the prestige or ranking of the Higher Education Institution of origin of the student is not a systematic factor of success for the student. Therefore, number of our students coming from Ethiopia (Mekele University), Indonesia (Bandug University) or from Bangladesh (Khulna University), ... that is to say coming from Universities/countries ignored in the Shanghai ranking list, succeeded as well as other students as long as their motivation, professional project and their studies background (especially the list of courses credits priorily obtained) correlate with our master program.

\section{TEACHING AND LEARNING METHODS}

The specificities of the CIMET Master also lie in the innovative pedagogical methods of teaching and learning and the Cross-European mobility scheme offered to students and teaching staff. Those two aspects are indeed enhancing the excellence and value of the Master program.

To stimulate student interest in learning and to encourage effective student training initiative several courses are based on a "project approach". For example, in 2010, in the Pattern Recognition course students had to propose a solution based on a machine learning approach to classify color objects of the PASCAL VOC database. The idea is to emphasis on independent learning and exploration of students, emphasize the development of self-learning abilities of students. The main objective is to exceed the traditional passive concept of learning by "learn" to "learn".

Each course of this master program is based on about 20 hours of lectures, 20 hours of exercises and 20 hours of practical laboratory sessions supervised by a teacher. Not counting the homework done by students. This is sufficient to introduce the main fundamentals of a subject but it is not enough to cover all aspects of a given item. To face this problem and to encourage active "self learning" teachers encourage students to read reference books, states of the art, scientific articles, etc. and guide students to find relevant information, algorithms and codes, on Internet. Teachers monitor student's self-construction of knowledge. To foster the open-mindness and the adaptability of students, several scientific seminars are proposed each semester on challenging issues.

That is, teaching theoretical courses that require the understanding of many mathematical models and equations to students who do not have a strong background in mathematics is always challenging. We had to face this problem especially with the color science course.
We have observed in this master program that the first half of a lecture on a theoretical subject is the most crucial part in terms of students' learning and feeling about the topic. We have observed that if a majority of students do not grasp the basics of a course during the first half of the lectures, a general lack of interest in the topic might be created. This lack of interest in one topic may even last for the entire semester and for any related topic during the following semester. It is even harder to find a solution to this problem when dealing with a heterogeneous student population with very different backgrounds and from very different academic systems. Identifying the students' learning style preferences and considering them in the design of learning and teaching packages can be a right solution. We have observed that a majority of students prefers: (1) seeing many graphs, graphics, images and relevant animations during the lecture; (2) testing concepts with interfaces, demos, practical test during the exercises/laboratory sessions; (3) that the teacher provides a great deal of verbal explanations during the lectures. We have also observed that a minority of students prefers having the opportunity to read a topic from a book or handbook, before a lecture or instead a lecture, and then ask the teacher any questions that they may have. The main question we tried to answer is "what is the optimum way to learn?" bearing in mind that studying in a master program is not the same thing as being in a magic show! Sometimes learning is not funny, so that does not make sense to try to answer to "how do they like to learn?" The questionnaires that we ask students to fill have shown that using other media, in addition to Powerpoint presentation and providing clear direction have the preference of students who do not seem to enjoy theoretical courses.

In the CIMET master, we tried to minimize the time taken in conveying the main message of each topic and to maximize their desire to learn and to some extent their enjoyment to learn. Thus, first we encouraged teachers to use multimedia systems in complement (not as a substitute) of traditional teaching/learning methods. In some courses videos, animations and menu items do not necessarily add value. We also encouraged teachers from consortium universities to use the same eWorking platform. Claroline is an Open Source eLearning and eWorking platform allowing teachers to build effective online courses and to manage learning and collaborative activities on the web. Each course dedicated space provides a list of tools enabling the teacher to: - publish documents in any format (text, PDF, HTML, video...), -develop learning paths, - create groups of students, - prepare online exercises, - propose assignments to be handed in online, use the wiki to write collaborative documents. We consider that eLearning courses are complementary to regular face-to-face courses. The aim is for students to be able to complete, add to and build on learning on specific subject, such as color science course which is based on a range of models and equations developed over the years, anywhere and anytime, i.e. outside the 
classroom whenever and for as long as they want. The idea is also to encourage students to do homework to deepen lectures content.

Unfortunately too many students think that programming is boring and prefer to use Mathlab language as this latter is widely used in engineering. Most of our students have a strong background in programming but not necessarily in algorithmic. Unfortunately, too many students think algorithmic is boring and useless, even for many students having a Bachelor in computer science. In order to increase the skills of our students in computer science and to do algorithmic much more appealing, rather than teaching them any specific programming language (e.g. $\mathrm{C}++$ or Java) we expose them in the Design and Analysis of Algorithms (DAA) course to a wide variety of pattern recognition or of image processing problems based on illustrative examples.

\section{A TRAINING PROGRAM COUPLED TO A PROJECT CONTEST}

In order to test the knowledge of CIMET students, to test their ability to face practical problems, to test their ability to confront open problems, to test their ability to suggest innovative ideas, to train them to work in groups with other students studying on other campuses, to train them to do their utmost to achieve a specific goal, a project contest is held each year. Students are divided in teams (at least four students per team). Teams are set in competition. They are confronted to the same project. The Group project is ongoing over the first three semesters of the program.

The idea is to emphasis on the innovative spirit of students and co-awareness. The project contest supervisor guides students discussion and provides appropriate comments and encouragement, pushes students to exchange different ideas, so that they mutually reinforce, and cultivate the enthusiasm of the students and also their sense of innovation and cooperation.

During the group project contest students have the chance to investigate theories and phenomena at the forefront of an applicative problem. Topic of the project work is defined every year separately. Thus, in 2008-2010, students had to face the issue of color image retrieval from images database. This first project contest was divided in four steps (e.g snapshot of color images, research of color invariants, features extraction, data analysis and processing) which cover fundamental courses taught during the first semester and compulsory courses taught during the second semester. Each team had also to carry out a fifth step. The content of this last step depended of the specializations chosen by students. The best solution was presented in CGIV conference in 2010. In 2009-2011, students had to face the issue of color image enhancement based on memory colors. The best solution will be presented in CCIW workshop in 2011.
Check points are organized each semester by the course instructor. All teams are evaluated at the end of the 3rd semester by the evaluation board. First, each group must submit a written project report. It must consist of chapters: Introduction, Methods, Experiments, Results, Discussion, and References. In addition, the software must be returned in digital form, to be tested by the evaluation board. 3 weeks after the final report submission, the groups present their project contest work and results as oral presentation. These presentations are organized by video conferencing. A price, sponsored by a company, is awarded each year to the best team. In 2010 the project contest was sponsored by Nokia.

\section{A LEARNING PROGRAM COUPLED TO A RESEARCH ACTIVITY}

After completing the Scientific Methodology course, the student shall be familiar with and shall be capable of discussing main questions and issues in scientific thinking. The student shall be able to frame research problems and questions, to develop a plan for conducting a scientific project and to report the results from scientific projects. The main goal for the course is to prepare the student for the upcoming master thesis and to be able to disseminate their scientific work through scientific publications.

CIMET students are strongly encouraged to conduct various research activities during their master in collaboration with research laboratories belonging to the consortium and are supported financially and scientifically in this activity.

The outlines of the Scientific Methodology course are:

1. Introduction to scientific research and scientific theory

2. What characterises good research

3. Ethics in research

4. Developing a research topic

5. Quantitative and qualitative research designs

6. State of the art and literature studies

7. Designing and analysing studies and experiments

8. Developing project plans

9. Conducting risk analysis and feasibility studies

10. Writing technical reports

The subjects of Master Thesis proposed to CIMET students contribute to stimulate the exchange of experience and know-how in this multidisciplinary domain. These subjects are defined to give Master's students the opportunity to obtain results of on-going research activities and to exchange opinions and experiences with research teams in an informal friendly environment. Position papers, progress reports on on-going projects and demos of running prototypes constitute the main form of communication offered by members of research teams. The expected spin-offs of the master thesis of the programme are for research teams: a) useful cross-fertilisation and b) formulation of joint pro- 
jects and research activities between European research teams.

Since the beginning of this master program in 2008 more than 20 scientific papers have been published by CIMET students. These papers have been presented in the following conferences: IEEE ICMV'2010, Hong Kong, China, IS\&T CGIV'2010, Joensuu, Finland, IEEE EUVIP'2010, Paris, France, IS\&T CIC'2010, San Antonio, USA, IEEE DICTA'2010, Sydney, Australia, EUSIPCO'2010, Aalborg, Denmark, IEEE IVCNZ'2010, Queenstown, New Zealand, AIC'2010 Mar del Plata, Argentina, CIS’2009, Gjøvik, Norway, SPIE Color Imaging'2009, San Jose, USA.

\section{PEPARING STUDENTS FOR THE GLOBAL LABOUR MARKET}

Another key element of this master program is to prepare students for the global labour market. The master program is therefore designed to address also industries' needs and challenges. Photonics \& Optical Technologies, Digital Imaging \& Computer Vision, Computer Science and Media Technologies are in full growth in all sectors of industrial developments. Indeed, the color element in these fields has an essential place. The needs are increasingly important and research in all these domains is dynamic. The results obtained by researchers worldwide are very quickly adapted for industry and technological purposes. The fields of teaching and learning of this master program are highly relevant to a wide range of sectors, as for example the photonic industry (e.g. lighting), the digital technology industry (e.g. display, printing), and all research and development activities involving computer science. The continued evolution of this research sector requires adapted and extremely specialized courses. On the international job market, the demand for Postgraduates in electronic imaging, color instrumentation, photonic and optical technologies as well as basic and applied research is very high. International companies such as Technicolor, UPM-Kymmene, Agfa-Gevaert, Hewlett Packard Corp., Xerox Corp., Nokia, Samsung, Philips, Sony as well as an increasing number of medium size businesses emerging on related technological fields need talented Postgraduate in those fields. Color science and optical technology are important technological areas for industrial activities in media creation, media processing and distribution, media reproduction and display. Laboratories and companies collaborating to the master CIMET gather large international academic and industrial contacts that can lead to various opportunities for our students. From research projects to full-time employment across Europe and beyond, contacts initiated within the Consortium are beneficial to all. In this master program students are strongly encouraged to do Summer Internship and Master Thesis in relation with Industry. Many industrial partners are involved in this master program in various ways: - education program (e.g. seminars), - visits to their companies, - research activity (e.g. project contest), funding of grants (e.g. summer internships, master thesis, PhD grants after completing the master), - quality board, - expert board, - contest project prize, - contract positions, etc.

\section{QUALITY ASSURANCE PROCEDURES}

Even if the four European universities involved in the master CIMET are all engaged in the Bologna process, it still exist a lot of differences between their educational systems. The main difficulty we faced at the beginning of the implementation of this master was to standardize our administrative procedures (exam scoring, master thesis scoring, resits consistency, etc.) and educational systems (diploma consistency, curriculum consistency, course consistency, prerequisite consistency, etc.). The main solution to face these issues is the flexibility offered by each partner.

In order to anticipate most of educational problems before the implementation of the master program, the objectives of the program had been clearly described in the course catalogue and in the learning agreement. The learning contents and objectives, the courses outlines, the learning outcomes and the assessment methods had been clearly defined. For each course, the learning objectives (aimed skills), the teaching methods, the course contents, the learning activities, the criteria and methods for assessment, etc. had also been clearly defined.

In order to face most of educational problems during the implementation of the master program an internal Quality Assurance, Evaluation and Supervision Board (QAESB) has been set up by the consortium. To ensure the quality of the learning and teaching processes and of the delivered diploma, the Joint Graduate Committee (JGC) and the QAESB have developed the following documents: - The Course Catalogue; - The Student Learning Agreement; - The transcript of Records and The Diploma Supplement.

The work of the QAESB is to regularly evaluate the quality of the individual teaching. The assessment is based on the analysis of online questionnaire filled out by all the students for each module, at the end of each module. The QAESB analyses all questionnaire results and makes recommendation to the local coordinators concerned to take the necessary measure to improve the course program. Results are made public and discussed with the students at the end of each year. This allows the program to improve and adapt as well as the tools for assessing the impact of quality assurance on learning.

The Quality Assurance, Evaluation and Supervision Board (QAESB) includes: - one representative member for each university of the consortium under proposal from each university; - two external members from other universities (of two other Erasmus Mundus pro- 
grams), companies (Technicolor, France \& Nokia, Finland).

Following the variety of the items, actions and protagonists to evaluate, the QAESB elaborate a proposal of standard processes, procedures and indicators to be used by all the universities of the consortium. In order to supervise the program, the QAESB determine: - the accurate procedures to evaluate the development and quality of the program; - the criterions and the actualization and improvement procedures of the program; the analysis procedures to evaluate the professional insertion of all the graduated from the Master and their satisfaction level for the received formation; - the mechanisms for the administrative management and the certification of the results, - the procedures for the evaluation of teachings and teachers.

The quality of the Master Program is also assured by the quality assurance procedures of all the universities of the consortium.

The master CIMET is an international master degree; in the cohort $2008-2010$ around $86 \%$ of students registered came from outside Europe, in the cohort 20092011 around 78\% of students registered came from outside Europe. In order to anticipate most of administrative problems (student's registration, student's accommodation, administrative procedures, etc.) and of individual problems (visa, culture shock, etc.) before and during the implementation of the master program, we have built up an International Student Handbook (ISH). This handbook has been considered as an example of good practice by the EACOVIROE (Enhance the Attractiveness of Computer Vision and Robotics in Europe) project [3]. For each university the handbook is structured as follow [4]:

- PART I :ABOUT UNIVERSITY

o Administrative procedure \& courses

o Being a student

o Facilities

- PART II : ADMINISTRATIVE PROCEDURES

o Visa \& Residence permits

o Financial Issues

o Health \& Insurance

- $\quad$ PART III : EVERYDAY LIFE

o Practical Information

o Useful information for your travel

o Everyday Life

o Facilities

o Emergencies

- PART IV : CULTURE, ENVIRONMENT \& WAY OF LIFE

o Culture

o Environnent

o General information

A key element of the marketing, recruitment, admission, support and management of excellent internation- al students is the availability, the dedication and the professionalism of coordination and international offices staff. The short and long term sustainability of such a master relies partly on the marketing strategy (e.g. study fairs, flyers, posters, website, mailing list, internet visibility, social networks such as Facebook, Twitter or LinkedIn, local, national or international articles, partnerships with companies, bilateral agreements with other Universities, scholarship forums etc..) developed by universities involved in the consortium.

\section{MANAGEMENT OF THE MASTER}

The Joint Graduate Committee (JGC) is the "core structure" in charge of the management of this mater program. This committee includes: - the academic coordinator; - the administrative coordinator; - the local coordinator of each partner university; - two external advisors from industries and - one students' representative.

This Committee meets at least three times a year to discuss the development of the course program, to discuss the QAESB recommendations and students' feedback, to select applicants and also for administrative issues (e.g. graduation ceremony, induction week) and exam-regulations. Additional meetings are also organized with the support of electronic multimedia (e.g. video-conference), especially for Master Thesis viva and some seminars. The availability and the dedication of JGC members (especially local coordinators) and the cooperation mechanisms within the consortium are two of the key factors of a successfully managed program.

The JGC is also responsible: - to create the conditions necessary for implementation of the program; - to control the procedures of the study program; - to guarantee that compulsory courses and optional courses are coherent with the Course Catalogue; - to decide upon syllabus changes; - to adjust re-sit and balance examination criteria within the curriculum; - to manage exceptions and problematic cases; - to deal with students under probation; - to allocate selected applicants (students and scholars) among the universities; - to pay attention to the principle of gender equality; - to change the conditions for the participation in the program if specific reasons require it; - to manage the tuition fees policy; to decide the amount of general costs kept by the coordinating institution; etc.

The JGC also relies on teaching staff members in each partner university, in order to guarantee the day-to-day running of the CIMET program, such as: - collection and monitoring of participating students curriculum; support the organization of common and compatible modules schedules; - preparation, collection and distribution of periodic reports; - processing and provision 
of students and scholars scholarships; - organization of periodical meetings.

Since the beginning of this master program the number of applicants increased exponentially: 2008: 80 applicants; 2009: 150 applicants; 2010: 125 applicants; 2011: 307 applicants. Here we only indicate the number of applicants that meet all requirements described in our application procedure. The three main reasons of this success are: - the promotion of the Erasmus Mundus programs by the EU; - the Master CIMET has become more and more recognized; - fields of global studies are becoming more and more popular.

Each year, the JGC has to face several issues, among them:

- some partner universities inside the consortium are more attractive than others (due to national language, living costs, climate, etc.), the JGC has therefore developed: - a policy towards students in order to have a roughly equal distribution of students among partners; and - a compensation system for "weak years".

- some students are more interested to do their Master Thesis in some partner universities (due to $\mathrm{PhD}$ opportunities, research facilities, etc.) or in some specialities rather than in others, the JGC has therefore developed: - a policy towards master thesis supervisors in order to encourage co-supervision of master thesis by two partner universities; - a policy towards students in order to have a roughly equal distribution of students among partners; - a compensation system for "weak years". In 2011 the consortium has proposed more than 60 topics of Master Thesis, among them more than 10 are in cosupervision with two partner universities and more than 10 are in collaboration with a company.

- most of our best students are more interested to do a PhD, after completion of their master, in US or in some EU countries rather than inside our consortium (due to salary, living cost, reputation, etc.), the JGC has implemented a policy in order to convince them that our laboratories offer other advantages (High Academic Standard, High Technological Environment, Modern/Innovative and creativity, Flexibility of study/research).

\section{CONCLUSION}

In this paper we have discussed several challenges that we tried to address in the international master course CIMET. For four years now, our aim has been to ensure excellence for our students. Such an aim is difficult to reach for many reasons. Some of them are highlighted in this article, others could be also described as the difficulties in everyday life being important when coordinating an international program involving several universities abroad. To face these difficulties the inevitable solution is to have a good quality assurance management process, a strong experience in innovative tools for teaching and learning, and the availability and dedication of academic partners, teaching staffs and administrative staffs.

\section{Bibliographie}

[1] Master Erasmus Mundus CIMET (Color in Informatics and Media Technology), http://www.mastererasmusmundus-color.eu/

[2] Erasmus Mundus Program, managed by the Education, Audiovisual and Culture Executive Agency (EACEA), http://eacea.ec.europa.eu/.

[3] EACOVIROE (Enhance the Attractiveness of Computer Vision and Robotics in Europe) project, http://www.eacoviroe.org.

[4] International Student Handbook (ISH), Master CIMET, http://www.master-erasmusmunduscolor.eu/content/view/line/22638. 